\title{
Commentary Oxytocin and the Social Brain: Beware the Complexity
}

\author{
Andreas Bartels $* 1,2$ \\ 'Vision and Cognition Lab, Centre for Integrative Neuroscience, University of Tübingen, Tübingen, Germany; ${ }^{2}$ Max Planck Institute for Biological \\ Cybernetics, Tübingen, Germany
}

Neuropsychopharmacology (2012) 37, 1795-1796; doi: I0.1038/npp.2012.7I

Love, or in more functional-biological terms, social attachment or bonding, is the evolutionary key to the existence of species like humans: our babies' survival depends entirely on parental care, which in turn provides the opportunity to transmit a vast amount of knowledge from one generation to the next. It is therefore no surprise that the brain's mechanisms that evolved to ensure parent-child bonding are powerful and under genetic control.

They involve the core of the reward system, where oxytocin (OT) and arginin-vasopressin (AVP) have been shown to be sufficient and necessary to induce parental attachment as well as pair-bonding, with highly consistent brain structures involved for both types of attachment in both human and animal (Bartels and Zeki, 2004; Young and Wang, 2004).

Recent studies have demonstrated dramatic effects of these neuropeptides in animals: a single dose of OT can induce a life-long pair-bond in prairie voles, or bring about the strong attachment of a mother to its child in virgin females to stranger pups (Young and Wang, 2004). Conversely, blocking signaling of OT or VP will prevent formation of pair-bonds and mother-child bonds, in the latter case leading to carelessly abandoned pups. In humans, similarly dramatic effects have been observed: for example, a single genetic variation of the brain's neuropeptide receptor gene can half the chances of getting married and double the frequency of relationship crises (Walum et al, 2008). The central role in our lives, but also translational aspects such as the emerging evidence for a close relation between mechanisms of attachment and addiction (McGregor and Bowen, 2012) - consider the symptoms of selfless devotion to the object of desire, followed by hopeless withdrawal - leave us bedazzled on why research on attachment mechanisms had been avoided for so long in psychology and later in neuroscience.

At present, human neuroimaging research on OT focuses primarily on its effects on generic social, mostly negatively valenced stimuli, such as faces with varying expressions, economical games involving risk and trust, negative

\footnotetext{
* Correspondence: Dr A Bartels, Vision and Cognition Lab, Centre for Integrative Neuroscience, University of Tübingen, Spemannstr. 38, Tübingen 72076, Germany. E-mail: andreas.bartels@tuebingen.mpg.de Received 10 April 2012; accepted 10 April 2012
}

conditioning or pain, and potential relations to disorders such as autism (Meyer-Lindenberg et al, 2011). Generally, these studies revealed a dampening effect of OT in regions that typically exhibit strong responses to negative stimuli, primarily in the amygdala. This evidence led to an interesting but perhaps premature 'all-dampening' view of OT action: OT attenuates neural responses to social cues in order to reduce social avoidance behavior and to facilitate social approach. Two more recent studies contradict this simple but attractive view: both showed amygdala upregulation with OT, one with positive facial expressions in males, the other with negative expressions in females (for a review, see Meyer-Lindenberg et al, 2011). Also, behavioral studies point to OT functions more complex than a mere cuddle-drug, as OT can also boost social exclusion, xenophobia, and selfishness (De Dreu et al, 2011), which, in monkeys, can reverse to prosocial behavior as a function of time after administration. Therefore, the neural action of OT in processing social cues may be more complex than 'all-dampening', in the amygdala and beyond, and may depend heavily on context (Bartz et al, 2011).

Given the past focus on generic social, frequently negatively valenced stimuli, the question arises about OT's effects in dopamine-responsive reward regions in the context of its core function - attachment to specific individuals. The present study of Wittfoth-Schardt et al. (2012) stands out as it sheds first light on this question.

The authors measured neural responses of fathers viewing images of their own, familiar, or unknown children. The study nicely extends analogous prior work and shows activation largely consistent with parts of the neural attachment system previously described in mothers and romantic partners (Bartels and Zeki, 2004). One region activated by viewing the own $v s$ a familiar child was the globus pallidus (GP), a key region belonging to the core of the reward and attachment systems, during placebo. OT attenuated this response and reduced GP's functional connectivity with several limbic regions. However, OT enhanced responses in another key region, the caudate nucleus, for the comparison of own child $v s$ unfamiliar child. This is the first demonstration of the modulatory effects - in both directions - of OT in context of its core function of attachment in the striatum and basal ganglia. 
Like all good studies, this one also opens up more questions than it answers. Particularly because of this, the authors' interpretation in terms of the previously suggested 'all-dampening' effect of OT to social stimuli, as a function of their salience, appears somewhat unsatisfactory: what functional or behavioral purpose would OT-mediated attenuation of reward responses to positive stimuli, in particular to the own child, have? Also, the observed OTinduced upregulation of caudate activity during own-child viewing is incompatible with this generic interpretation.

The results ask for different, novel, more radical interpretations, but also for patience, as more data and studies like this are needed. Strikingly, the present data show that the strongest neural effects of OT administration-in terms of magnitude and number of regions involved-concerned the contrast of viewing unfamiliar children $v s$ familiar children, with seven downmodulated regions, in contrast to only one down- and one upmodulated region for contrasts involving the own child $v s$ other children. The only coinciding region was the GP, which also differentiated between own and unknown child, changing its connectivity only in the OT interaction with the own child, but not with the unfamiliar child.

These results suggest that OT action is highly specific to the exact type of the social stimulus viewed, and to the network activated by the stimulus. Viewing of unknown children activated networks of critical social assessment, also involved in making judgments on trustworthiness during placebo, which were attenuated by OT. This part of the results fits the dampening interpretation of OT that facilitates approach toward the unknown.

In contrast, viewing the own child leads to activity in reward-related regions, with no automatic neural avoidance reaction that could or should be dampened. On the contrary, it is the stimulus-induced activation of the reward regions that is thought to help promote prosocial behavior toward the own child, and to facilitate potentially aversive actions, such as changing nappies, or altruistic and demanding behavior such as nurturing. In contrast to most other social stimuli, neural attachment responses are a consequence of lasting structural changes in the reward centers that were originally caused by OT and AVP (Young and Wang, 2004). To complicate matters, viewing the own child may inherently lead to OT release in the brain - additional application of OT may therefore partly lead to autoregulatory action. Alternatively, effects of intranasal OT during viewing of attachment stimuli may be relatively small because of OT presence due to internal OT release also in the placebo condition, but not during viewing of nonattachment stimuli.
The most interesting aspect may lie in considering the power of OT to induce plasticity in the reward system: interactions of OT with attachment stimuli may provide a glimpse into the mechanisms involved in maintaining longterm attachment - $a$ hitherto unexamined territory in attachment research, both of the romantic and of the parental type. This also has potentially important consequences for translational approaches of attachment research: as plasticity allows for change, the interaction of OT with reward-processing regions may provide alternate insights into treatments of social disorders such as autism (MeyerLindenberg et al, 2011), or of addiction to substances rather than to human beings (McGregor and Bowen, 2012).

In the future, the real challenge may be twofold: first, in order to bridge the gap from neuroimaging to mechanistic understanding, corresponding invasive animal work is necessary. Second, we need to find clever ways to present social stimuli in truly social context if we want to understand the complex ways in which OT shapes the human nature.

\section{DISCLOSURE}

The author declares no conflict of interest.

\section{REFERENCES}

Bartels A, Zeki S (2004). The neural correlates of maternal and romantic love. Neuroimage 21: 1155-1166.

Bartz JA, Zaki J, Bolger N, Ochsner KN (2011). Social effects of oxytocin in humans: context and person matter. Trends Cogn Sci 15: 301-309.

De Dreu CK, Greer LL, Van Kleef GA, Shalvi S, Handgraaf MJ (2011). Oxytocin promotes human ethnocentrism. Proc Natl Acad Sci USA 108: 1262-1266.

McGregor IS, Bowen MT (2012). Breaking the loop: oxytocin as a potential treatment for drug addiction. Horm Behav 61: 331-339.

Meyer-Lindenberg A, Domes G, Kirsch P, Heinrichs M (2011). Oxytocin and vasopressin in the human brain: social neuropeptides for translational medicine. Nat Rev Neurosci 12: 524-538.

Walum H, Westberg L, Henningsson S, Neiderhiser JM, Reiss D, Igl W et al (2008). Genetic variation in the vasopressin receptor 1a gene (AVPR1A) associates with pair-bonding behavior in humans. Proc Natl Acad Sci USA 105: 14153-14156.

Wittfoth-Schardt D, Grunding J, Wittfoth $M$, Lanfermann $H$, Heinrichs M, Domes G et al (2012). Oxytocin modulates neural reactivity to children's faces as a function of social salience. Neuropsychopharmacology 5: 6-9.

Young LJ, Wang Z (2004). The neurobiology of pair bonding. Nat Neurosci 7: 1048-1054. 